\title{
Conocimiento de las madres sobre la diarrea y su prevención en un asentamiento humano de la provincia de Ica, Perú
}

\author{
J. Jhonnel Alarco ${ }^{a}$, Eduardo Aguirre-Cuadros ${ }^{a}$, \\ Esmilsinia V. Alvarez-Andrade ${ }^{a}$
}

aEstudiante de Medicina, Facultad de Medicina, Universidad Nacional San Luis Gonzaga. Ica, Perú.

Correspondencia: J. Jhonnel Alarco, Santa Rosa del Palmar X-25 Ica, Perú. Correo electrónico: jhonnelalarco@ gmail.com

Recibido el 26 de Septiembre de 2012

Aceptado para su publicación el 1 de Mayo de 2013.

\section{RESUMEN}

Objetivo: Determinar el nivel de conocimiento de las madres sobre la diarrea y su prevención en un asentamiento humano de la provincia de Ica, Perú.

Diseño: Estudio observacional, descriptivo de corte transversal.

Emplazamiento: Asentamiento humano "El Huarango", ubicado a $2 \mathrm{~km}$ de la ciudad de Ica, población en extrema pobreza como producto de un desastre natural.

Participantes: 82 madres de niños menores de 5 años.

Mediciones principales: Se evaluó el nivel de conocimiento sobre la diarrea y su prevención a través de un cuestionario elaborado por expertos en el tema y validado en la misma población, previo a su aplicación.

Resultados: Se entrevistaron a 82 madres, el promedio de edad fue de 29 años (con una mínima de 15 y una máxima de 49 ). El 58,5\% de las madres fueron convivientes, el $73,1 \%$ solo se dedicaban a los quehaceres domésticos (ama de casa), alrededor del $7 \%$ tenían estudios superiores culminados y solo el $6 \%$ de las viviendas contaban con el servicio de desagüe. Sobre los conocimientos y prevención de la diarrea, el $76 \%$ conoció el significado de la palabra diarrea, el $53 \%$ creyeron que los parásitos son la principal causa, el $53 \%$ prefirió usar el suero de rehidratación oral (SRO) como principal medida de tratamiento, aunque un $23 \%$ admitió que le daría antibióticos, el $67 \%$ acudiría al centro de salud, mientras que un $24 \%$ lo haría al curandero. Conclusiones: El conocimiento general de las madres sobre el manejo de la diarrea, es regular, persiste la creencia de que se debe de prohibir la lactancia materna, de que los parásitos son principal causa y que se debe usar antibióticos como parte de un tratamiento adecuado.

Palabras clave: Atención Primaria de Salud, Educación en Salud, Áreas de pobreza, Madres, Diarrea, Perú.

\section{ABSTRACT}

\section{Objective:}

To determine mothers' knowledge of diarrhoea and its prevention in a human settlement in the province of Ica, Peru

Design: Cross sectional observational descriptive-type study

Location: "El Huarango" human settlement situated $2 \mathrm{~km}$ from the city of Ica, a city in a situation of extreme poverty caused by a natural disaster.

Participants: 82 mothers of children under 5 years of age.

Main Surveyed Data: Knowledge of diarrhoea and its prevention was assessed via a questionnaire prepared by experts in the subject and validated by the population, prior to implementation.

Results: 82 mothers were interviewed. Their average age was 29 (with a minimum age of 15 and a maximum of 49 ). $58.8 \%$ of the mothers were live-in partners, $73.1 \%$ were housekeepers, approximately $7 \%$ had completed higher eduction and only $6 \%$ of the dwellings had drainage systems. With regard to knowledge and prevention of diarrhoea, $76 \%$ knew the meaning of the word diarrhoea, $53 \%$ believed parasites to be the main cause and $53 \%$ preferred to use oral rehydration solution (ORS) as the main treatment measure, although $23 \%$ admitted they would give antibiotics. $67 \%$ would go to a health centre, whereas $24 \%$ would go to a medicine man (healer).

Conclusions: The mothers' general knowledge of how to deal with diarrhoea is mediocre. The belief that breastfeeding should be forbidden persists, that parasites are the main cause and that antibiotics should be used as an appropriate part of treatment.

Keywords: Primary health care, Health education, Areas of poverty, Mothers, Diarrhoea, Peru. 


\section{INTRODUCCIÓN}

La Organización Mundial de la Salud define a la diarrea como la presencia de tres o más deposiciones al día o una frecuencia mayor que la normal, de heces sueltas o líquidas. La infección puede ser causada por bacterias, virus $\mathrm{y} / \mathrm{o}$ parásitos y se transmite por alimentos o agua de consumo contaminado, así como de una persona a otra como resultado de una higiene deficiente ${ }^{1,2}$.

Las enfermedades diarreicas agudas (EDAs), a nivel mundial, representan la segunda mayor causa de mortalidad en niños menores de cinco años, además afectan a todos los grupos etarios, pero los más afectados son los niños. En algunas zonas del Perú se ha encontrado que la incidencia de enfermedad diarreica es de 4,38 episodios por niño-año, siendo muy superior al promedio mundial ${ }^{2,3}$.

En el Perú, las EDAs son responsables del 7\% de la mortalidad de niños menores de 5 años de edad y son uno de los principales motivos de consulta médica ${ }^{4}$. Del total de hospitalizados por EDAs en el año 2010, más de $50 \%$ fueron menores de 5 años. Para el año 2011, hasta la semana epidemiológica (SE) 04 , el $51 \%$ de los episodios de EDAs notificados fueron en menores de 5 años, mostrando una tendencia similar al periodo $2010^{5}$.

En la región Ica en el año 2009, según su Dirección Regional de Salud (DIRESA-Ica) las diarreas ocupan el tercer lugar en atenciones por consulta externa en todos los establecimientos de salud con 33.812 casos, en ese mismo periodo las enfermedades infecciosas y parasitarias son la primera causa de enfermedad en la región con 256.402 atenciones, representando el $44.97 \%$ del total ${ }^{6}$.

El asentamiento humano "El Huarango", sector de Tierra Prometida agrupa a 250 familias que viven en condiciones de extrema pobreza, en una zona ubicada a $2 \mathrm{~km}$. al oeste de la ciudad de Ica, en pleno desierto costero, cuyos orígenes se remontan a una inundación ocurrida el año 1998, producto del desborde de su cauce, del rio Ica. Como consecuencia, cerca de 402 familias llegaron a dicha zona buscando resguardo. Divididos en 3 grupos, decidieron establecerse y empezar de nuevo, soportando duras condiciones de vida, como las altas temperaturas que en verano llegan hasta $50^{\circ} \mathrm{C}$ dentro de las viviendas.

Muchos procesos morbosos y defunciones se evitan cuando las madres dominan, cumplen y propician buenas costumbres higiénicas en la familia ${ }^{7}$. Por esta razón decidimos valorar el nivel de conocimiento de las progenitoras en el asentamiento humano "El Huarango", del distrito de Ica, con la finalidad de contribuir a su perfeccionamiento e implementar estilos de vida saludables, por ser ésta una población vulnerable debido a un deficiente saneamiento ambiental, poca accesibilidad a los servicios de salud, nivel socioeconómico bajo, infraestructura educativa deficiente y condiciones climáticas extremas.

\section{MATERIAL Y MÉTODOS}

Se realizó un estudio observacional, descriptivo de corte transversal para evaluar el conocimiento sobre la enfermedad diarreica y su prevención en 82 madres de niños menores de 5 años, pertenecientes al asentamiento humano "El Huarango", durante el período comprendido de mayo a junio del 2011.

La población alberga aproximadamente cerca de 100 familias. Realizamos un muestreo de tipo no probabilístico. Los criterios de inclusión fueron: ser madre y tener un niño menor de cinco años y ser residente permanente del asentamiento humano. Los criterios de exclusión fueron: tener alguna patología psiquiátrica 0 de déficit mental que pudiera dificultar la interpretación de las preguntas y no ser residente permanente.

Esta investigación se llevó a cabo como parte de las campañas de proyección a la comunidad realizadas por la facultad de medicina "Daniel Alcides Carrión", de la Universidad Nacional "San Luis Gonzaga" de Ica.

Se utilizó, como instrumento de investigación, un cuestionario con las variables de interés para la investigación dividido en dos partes:

A. Datos generales de la población estudiada: estado civil, lugar de procedencia, grado de instrucción, ocupación de la madre, tipo de vivienda y servicios básicos (6 preguntas).

B. Conocimientos sobre el manejo y prevención de la diarrea: concepto, causas, signos de deshidratación, restricción de alimentos, tratamiento y acción a tomar en caso se presentase el cuadro diarreico (6 preguntas).

Estas últimas preguntas fueron de opción múltiple, con solo una respuesta correcta. El grado de conocimiento se dividió en tres categorías: bueno (si tenía 5 ó 6 respuestas correctas); moderado (si tenía 3 ó 4 respuestas correctas) y malo (si tenía 1 ó 2 respuestas correctas). El instrumento fue aplicado por los responsables de la investigación. Se solicitó a las madres participantes del estudio su consentimiento informado y escrito.

El instrumento fue validado a través de la revisión de sus contenidos por profesionales especialistas en el tema y por la aplicación de la prueba piloto al $10 \%$ de la población (10 madres).

Para la cuantificación de los datos se utilizó el programa Microsoft Excel 2010 y como medida de resumen para los datos estadísticos obtenidos se utilizaron medidas de tendencia central: cantidades, promedios y porcentajes.

\section{RESULTADOS}

Las principales características de la población estudiada se resumen en la tabla 1. 


\begin{tabular}{|c|c|c|}
\hline Variables & $\mathbf{N}$ & $\%$ \\
\hline \multicolumn{3}{|l|}{ Estado civil de la madre } \\
\hline Soltera & 13 & 15,8 \\
\hline Casada & 14 & 18,3 \\
\hline Divorciada & 2 & 2,4 \\
\hline Viuda & 5 & 6,9 \\
\hline Conviviente & 48 & 58,5 \\
\hline \multicolumn{3}{|l|}{ Lugar de procedencia de la madre } \\
\hline Ayacucho & 12 & 14,6 \\
\hline Ica & 54 & 65,9 \\
\hline Lima & 4 & 4,9 \\
\hline Otros departamentos & 12 & 14,6 \\
\hline \multicolumn{3}{|l|}{ Grado de instrucción de la madre } \\
\hline Primaria completa & 8 & 9,8 \\
\hline Primaria incompleta & 4 & 4,9 \\
\hline Secundaria completa & 32 & 39,0 \\
\hline Secundaria incompleta & 22 & 26,8 \\
\hline Superior completa (Instituto o universidad) & 6 & 7,3 \\
\hline Superior incompleta & 10 & 12,2 \\
\hline \multicolumn{3}{|l|}{ Ocupación de la madre } \\
\hline Ama de casa & 50 & 73,1 \\
\hline Trabaja & 18 & 21,9 \\
\hline Estudiante & 2 & 2,4 \\
\hline Ninguno & 2 & 2,4 \\
\hline \multicolumn{3}{|l|}{ Tipo de vivienda } \\
\hline Cemento/ladrillo & 12 & 14,6 \\
\hline Madera & 56 & 68,3 \\
\hline Quincha, adobe & 14 & 17,1 \\
\hline \multicolumn{3}{|l|}{ Qué servicios tiene su hogar } \\
\hline Luz & 70 & 85,3 \\
\hline Agua & 72 & 87,8 \\
\hline Desagüe & 6 & 7,3 \\
\hline Silo & 36 & 43,9 \\
\hline Ninguno & 4 & 4,9 \\
\hline
\end{tabular}

Tabla 1. Características de la población estudiada.

La edad mínima de las madres fue de 15 años y la máxima de 49 años, con un promedio de edad de 29 años.

El nivel de conocimiento de las madres participantes en el estudio se muestra en las siguientes tablas:

En la tabla 2 se observa que el concepto correcto de heces liquidas y aumento en el número de deposiciones se impone como respuesta correcta sobre las demás alternativas con un $75.6 \%$.

En la tabla 3 se describe a los parásitos como la principal causa de diarrea con un $50.0 \%$, seguido de la falta de higiene con un $34.1 \%$. 
En la tabla 4 se afirma que el dolor abdominal es un signo de deshidratación (39.0\%), seguido de la sed aumentada con $35.4 \%$.

Las madres afirman que durante la diarrea no se debe restringir ningún alimento $(36.6 \%)$, sin embargo, aún persiste la creencia de que la leche materna se debe suspender con un $30.4 \%$.

En la tabla 6 se afirma que el $53.3 \%$ considera la administración de Suero de Rehidratación Oral (SRO) como la medida más adecuada a tomar en caso de diarrea, aunque persiste la intención de dar antibióticos (automedicación) en un 23.2\%.

La acción a tomar, en caso de diarrea, se describe en la tabla 7. Destaca la intención de acudir al centro de salud con un $67,0 \%$, sin embargo, persiste la idea de llevar al curandero con un 24,4\%.

El conocimiento general de las madres sobre la diarrea es regular en una mayor proporción $(42,7 \%)$, seguido de un conocimiento calificado como bueno $(34,1 \%)$ y calificado como malo, con un $23.2 \%$.

\begin{tabular}{lcc}
\hline \multicolumn{1}{c}{ Significado de la diarrea } & N & \% \\
\hline Heces liquidas y aumento del número de deposiciones & 62 & 75.6 \\
Heces blanquecinas y malolientes & 9 & 10.9 \\
Dolor de barriga & 5 & 6.2 \\
Estreñimiento & 4 & 4.9 \\
Desconoce & 2 & 2.4 \\
\hline
\end{tabular}

Tabla 2. Conocimientos de las madres sobre el significado de la diarrea.

\begin{tabular}{lccc}
\hline & Principal causa de la diarrea & N & $\%$ \\
\hline Falta de higiene & 28 & 34.1 \\
Parásitos & 41 & 50.0 \\
Empacho por alimentos & 5 & 6.1 \\
Crianza de animales & 6 & 7.3 \\
Desconoce & 2 & 2.4 \\
\hline
\end{tabular}

Tabla 3. Conocimientos de las madres sobre las causas de diarrea.

\begin{tabular}{lcc}
\hline \multicolumn{1}{c}{ Conocimientos sobre los signos de deshidratación } & N & $\%$ \\
\hline Sed aumentada & 29 & 35.4 \\
Dolor abdominal & 32 & 39.0 \\
Dolor de cabeza & 16 & 12.2 \\
Tos & 0 & 46.3 \\
Desconoce & 6 & 7.3 \\
\hline
\end{tabular}

Tabla 4. Conocimientos de las madres sobre los signos de deshidratación. 


\begin{tabular}{|c|c|c|}
\hline Restricción de los alimentos en la diarrea & $\mathbf{N}$ & $\%$ \\
\hline Ninguno & 30 & 36.6 \\
\hline Carne & 6 & 7.3 \\
\hline Pescado & 10 & 12.2 \\
\hline Leche materna & 25 & 30.4 \\
\hline Desconoce & 11 & 13.4 \\
\hline
\end{tabular}

Tabla 5. Conocimientos de las madres sobre la restricción de alimentos durante la diarrea.

\begin{tabular}{|c|c|c|}
\hline Medidas adecuadas en caso de diarrea & $\mathbf{N}$ & $\%$ \\
\hline Suero de Rehidratación Oral (SRO) & 47 & 53.3 \\
\hline Café & 1 & 1.2 \\
\hline $\mathrm{Te}$ & 14 & 17.1 \\
\hline Antibióticos & 19 & 23.2 \\
\hline Desconoce & 1 & 1.2 \\
\hline
\end{tabular}

Tabla 6. Conocimientos de las madres sobre las medidas adecuadas en caso de diarrea.

\begin{tabular}{lcc}
\hline \multicolumn{1}{c}{ Acción a tomar en caso de diarrea } & N & $\%$ \\
\hline Llevar al Centro de Salud & 55 & 67,0 \\
Acudir al curandero & 20 & 24,4 \\
Dar medicación & 6 & 7,3 \\
Esperar que se recupere solo & 1 & 1,2 \\
Desconoce & 0 & 0,0 \\
\hline
\end{tabular}

Tabla 7. Conocimientos de las madres sobre la acción a tomar en caso de diarrea.

\begin{tabular}{lccc}
\hline & Nivel de conocimiento general & $\mathrm{N}$ & $\%$ \\
\hline Bueno & 28 & 34,1 \\
Regular & 35 & 42,7 \\
Malo & 19 & 23,2 \\
\hline
\end{tabular}

Tabla 8. Conocimiento general de las madres sobre el manejo de la diarrea en niños menores de 5 años. 


\section{DISCUSIÓN}

El moderado conocimiento sobre el manejo de la diarrea en madres del asentamiento humano "El Huarango", podría tener relación con la elevada prevalencia de enteroparasitosis descrito en los registros del puesto de salud de la comunidad (datos no publicados).

Se han realizado numerosos estudios sobre el conocimiento y prevención de las diarreas, ya sea en madres de familia, cuidadores de infantes o en el personal sanitario.

El $76 \%$ de la población en estudio tenía correctamente definido el concepto de diarrea, mientras que la mitad de la población creía que la principal causa de la diarrea era por contagio con parásitos. Un estudio realizado en $\mathrm{Cuba}^{7}$ tuvo resultados similares, la población aceptaba erróneamente que el parasitismo era la principal causa, seguido de la falta de higiene, cuando realmente es al contrario.

En este mismo estudio ${ }^{7}$ se destacó, que el manejo adecuado de la diarrea se daba por administrar suero de rehidratación oral (SRO) en un 78.4\%, cifra que podemos considerar favorable. En una investigación realizada en la India ${ }^{11}$ se encontró que las madres preferían usar SRO en un 65\%, cifra que luego de una intervención sanitaria y nutricional aumentó a 98\%; sin embargo, estas cifras son altas si lo comparamos con el 53,3\% que reportamos en este estudio, lo que demuestra la falta de conocimiento sobre el adecuado uso de SRO y la necesidad de realizar intervenciones de promoción y prevención de la salud.

El $23,2 \%$ de la población encuestada tenía la idea errónea de que la diarrea debía ser tratada con antibióticos, cifra preocupante por el riesgo de que una gran cantidad de progenitoras puedan recurrir a la automedicación antes de acudir al centro de salud. Esta proporción es baja si lo comparamos con el estudio de Ahmed y col. $^{8}$ realizado en Cachemira (India) donde se encontró que el 77,9\% de las madres tenían preferencias por el uso de antibióticos antes que otro tratamiento. En un estudio similar realizado en México, se encontró un porcentaje de $10.2 \%$ de preferencia, incluso se detalla que las madres medicaban a sus hijos con Sulfametozaxol y Trimetropina antes de acudir al centro de salud ${ }^{9}$.

Estos datos sobre la preferencia del uso de antibióticos son propias de poblaciones con bajos recursos y de zonas rurales; sin embargo, un estudio realizado en Arabia Saudita en una población urbana demostró esta preferencia en un 25\% de la población, mientras que la opción de usar SRO no fue mencionada por ningún entrevistado ${ }^{12}$.

El $36.6 \%$ creía que no se debe prohibir ningún alimento durante un cuadro diarreico. Este concepto es correcto; sin embargo, un $30.4 \%$ sostenía la idea de prohibir la lactancia materna por considerarla perjudicial para el manejo de la diarrea, sin saber que el amamantamiento reduce significativamente la cantidad y frecuencia de las evacuaciones, a la vez que garantiza una recuperación más rápida del afectado ${ }^{12}$. Nuestros hallazgos son similares al estudio de Pahwa y col. ${ }^{11}$, donde las madres creían que la leche materna era saludable durante un episodio de diarrea, solo en un $46.7 \%$.

Acudir al centro de salud frente a un cuadro diarreico fue la opción correcta en el $67 \%$ de la población estudiada, mientras que el $24.4 \%$ tenía la intención de buscar al curandero. Esta cifra es superior al estudio de Shah y col. ${ }^{10}$, realizado en la India, donde se reportó que la voluntad de acudir a un curandero o chaman fue del $10.9 \%$. Similares datos fueron mostrados por Bella y col. ${ }^{12}$, donde un $92 \%$ de los encuestados no estaban dispuestos a enviar a sus hijos ver a un "doctor", sino que eran tratados en casa o se buscaba la asistencia de un santero.

Si bien nuestra investigación solo pretendió medir el nivel de conocimiento de las madres en un momento determinado, encontramos ciertas circunstancias que no estuvieron planteados en el diseño del estudio y que no podemos dejar de mencionar. Encontramos que las madres tenían la creencia de que el uso de SRO podía curar la diarrea, por lo que no llevaban a sus hijos al puesto de salud, lo que sería extremadamente perjudicial para el niño. Este hallazgo no pudo ser cuantificado, por lo que sugerimos su inclusión en futuras investigaciones.

\section{CONCLUSIONES}

Según nuestro estudio el nivel de conocimiento sobre la diarrea, en las madres del asentamiento humano "El Huarango", es regular; persiste la creencia de que se debe prohibir la lactancia materna en caso de un cuadro diarreico, de que los parásitos son la principal causa y que se deben usar antibióticos como parte de un tratamiento adecuado.

Es importante reconocer el papel que cumplen las instituciones académicas, que fomentan la realización de campañas de proyección a la comunidad, donde los estudiantes tenemos la oportunidad de participar y estar en contacto con poblaciones en extrema pobreza, con necesidades básicas insatisfechas (vivienda, servicios sanitarios, educación e ingreso mínimo), que adolecen de servicios médicos adecuados, donde es indispensable la realización de campañas de prevención y promoción de la salud. A su vez, estas actividades deben ser reforzadas por los medios de difusión masiva (radio, televisión, periódicos regionales, etc.), para que luego se amplíen y debatan en la comunidad con participación activa 
de los profesionales en atención primaria y los estudiantes de ciencias de la salud.

\section{AGRADECIMIENTOS}

Los autores agradecemos a los pobladores del asentamiento humano "El Huarango", por las facilidades prestadas en la realización de este estudio.

\section{BIBLIOGRAFÍA}

1. Organización Mundial de la Salud. Enfermedades Diarreicas. Nota descriptiva $\mathrm{N}^{\circ} 330 ; 2009$. disponible en: http://www.who.int/mediacentre/factsheets/fs330/es/ print.html.

2. Miranda J, Ramos W. Pronóstico de la tendencia nacional y regional de las enfermedades diarreicas agudas en menores de cinco años de edad en el Perú mediante un modelo ARIMA con el enfoque Box-Jenkins. Revista Peruana de Epidemiología 2010(1):24-31.

3. Guillén A. Enfermedad diarreica: un problema recurrente de salud pública. Rev Peru Med Exp Salud Publica 2011;28(1).

4. Perú, Presidencia del Consejo de Ministros. Resumen Ejecutivo. Informe del cumplimiento de los objetivos de desarrollo del milenio. Lima: Comisión Interministerial de Asuntos Sociales CIAS; 2009.

5. Venero EG, Cruz AR. Enfermedad Diarreica Aguda. Bol Epidemiol (Lima) 2009; 18:992-3.
6. Siguas Cortez JS. Vigilancia epidemiológica con posteridad a desastres naturales en las provincias afectadas por el sismos realizada por la DIRESA Ica. 2009.

7. Cervantes Baute IC, Bosch Govea M, Armero Pérez G. Valoración del conocimiento de las madres sobre las diarreas y su prevención. Revista Cubana de Enfermería 2001; 17(1): 56-59.

8. Ahmed F, Farheen A, Ali I, Thakur M, Muzaffar A, Samina M. Management of Diarrhea in Under-fives at Home and Health Facilities in Kashmir. International Journal of Health Sciences 2009;3(2):171.

9. Alzate-Ávila G, Vázquez-Uribe C, Rojas-Montoya CC, Salcedo-Parra AE, López-Mariscal N, Quezada-López MS. Automedicación y prácticas empíricas de la madre de niños menores de 5 años que presentan enfermedad diarreica infecciosa. Humanitatis. 2007; 10(1): 118-122.

10. Shah MS, Ahmad A, Khalique N, Afzal S, Ansari MA, Khan Z. Home-based management of acute diarrhoeal disease in an urban slum of Aligarh, India. The Journal of Infection in Developing Countries 2011;6(02):137-142.

11. Pahwa S, Kumar GT, Toteja GS. Performance of a community-based health and nutrition-education intervention in the management of diarrhoea in a slum of Delhi, India. J Health Popul Nutr 2010; 28(6):553-9.

12. Bella $\mathrm{H}$, Al-Freihi $\mathrm{H}$, El-Mousan $\mathrm{M}$, Twum Danso $\mathrm{K}$, Sohaibani M, Khazindar S. Knowledge, Attitudes and Practices related to Diarrhoea in Eastern Province, Saudi Arabia. J Med Familia y Comunidad. 1994; 1 (1): 40-44. 